\title{
EVALUATION OF MACHINE LEARNING TECHNIQUES IN VINE LEAVES DISEASE DETECTION: A PRELIMINARY CASE STUDY ON FLAVESCENCE DORÉE
}

\author{
Jonáš Hruška ${ }^{1}$, Telmo Adão ${ }^{1,2}$, Luís Pádua ${ }^{1,2}$, Nathalie Guimarães ${ }^{1}$, Emanuel Peres ${ }^{1,2}$, Raul Morais ${ }^{1,2}$ and Joaquim João Sousa ${ }^{1,2}$ \\ ${ }^{1}$ University of Trás-os-Montes e Alto Douro, 5000-801 Vila Real, Portugal - (jonash,nsguimaraes)@utad.pt \\ ${ }^{2}$ INESC TEC Technology and Science, 4200-465 Porto, Portugal - (telmoadao,luispadua,eperes,rmorais,jjsousa)@utad.pt
}

\author{
Commission VI, WG VI/4
}

KEY WORDS: Disease detection, Flavescence Dorée, Hyperspectral data, Machine learning, Viticulture

\begin{abstract}
:
Vine culture is influenced by many factors, such as the weather, soil or topography, which are triggers to phytosanitary issues. Among them are some diseases, that are responsible for major economic losses that can, however, be managed with timely interventions in the field, viable of leading to effective results by preventing damage propagation. While not all symptoms might present a visible evidence, hyperspectral sensors can tackle this aspect with their ability for measuring hundreds of continuously sparse bands that range beyond the eye-perceptible spectrum. Having such research line in mind in this work, a hyperspectral sensor was applied to analyse the spectral status of vine leaves samples, collected in three chronologically distinct campaigns, while costly and destructive laboratory methods were used to track Flavescence Dorée (FD) in the same samples, for a ground truth information. Regarding data processing, machine learning approaches were used, in which several classifiers were selected to detect FD in vine leaves hyperspectral images. The goal was to evaluate and find most suitable classifier for this task.
\end{abstract}

\section{INTRODUCTION}

The sustainable production of vines envisages challenges induced by many factors - such as the weather, soil or topography - that are hard to control, from which can be highlighted phytosanitary issues in general and diseases in particular, responsible for major economic losses in the Agriculture industry worldwide (Martinelli et al. 2015). In 1990s, new discipline called Precision viticulture was developed. It aims to adjust vineyard management to the spatial variability that is present in the field in order to make its economic and environmental sustainability more efficient (Santesteban 2019). Disease management usually involve inadequate plant protection products (PPPs) administration, due to the lack of proper detection techniques and decision support systems capable of identifying affected areas and quantifying the real needs, in a timely manner. In fact, the well-timed detection would have an positive effect for optimal diseases control actions and plant growth management strategies (Akila, Deepan, 2018). Savary et al. (2012) also pointed out in their study that early detections increases treatments effectiveness. In an ecological perspective, PPPs management aims to reduce negative environmental impacts, as well. The study of (Popp et al., 2013) demonstrates the real consequences of unmanaged pesticide spraying products on consumers health. The traditional in-field visual observation by an agronomist or other similar professional and related laboratory test are only practicable for small areas because it is very time consuming and demanding. Moreover, the subjectivity of visually identifying phyto-pathological problems can lead to misclassification and wrong conclusions even by experienced agricultural experts (Akila, Deepan 2018). Nevertheless, available non-invasive optical sensors that can access to reflectance properties of a plants in wide range of the electromagnetic spectrum in narrow bands has been emerging, with the potential for more effective disease detection (Chen et al., 2019; Lowe et al., 2017; Mahlein et al., 2012; Thomas et al., 2017; Xie et al.,2015; Zhou et al., 2019).

Among them, hyperspectral sensors can be highlighted, whose principle is comparable to the one behind common RGB or multispectral cameras, i.e., measuring the amount of light that reach the sensor and store their formation. However, they differ in the range of electromagnetic spectrum they can capture, as well as in the number and width of the bands associated. RGB camera only measures three wide bands in the visual part of spectrum, multispectral typically from tree to fifteen bands, while hyperspectral camera measures up to several hundreds of narrow bands ranging much more than the eye can meet. Besides, it is known that when symptoms become visible, in most cases, disease development already reached a middle or late stage (Lowe et al, 2017). On the other hand, hyperspectral image - also known as hyperspectral cube - contains two dimensions: spatial and spectral information. (Thomas et al. 2017). The detailed spectral information in hyperspectral images allows an association to physiological processes in plants. Hyperspectral reflectance pattern for such characterization was already validated along with destructive methods in previous works (e.g. determination of photo pigments (Zhao et al., 2016)). Furthermore, hyperspectral imaging is an objective method in contrast to visual interpretation which makes it suitable for implementation in automated systems (Mahlein, 2015; Virlet et al., 2017; Walter et al., 2015).

The hyperspectral imaging can be used for a different scale application. In the work carried out by Thomas et al. (2017), the scales are divided into tissue, leaf, single plant and canopy. Tissue is the most detailed scale with resolution in millimetres; leaf scale operates with centimetres resolution. Both of those scales are commonly acquired in laboratory environment. Plant scale is defined up to $30 \mathrm{~cm}$ and canopy up to $50 \mathrm{~cm}$ resolution. However, even bigger scales can be considered, such as field and landscape, namely while resorting to hyperspectral sensor- 
capable UAV, airplane or satellite (Adão et al. 2017). For each application the trade-off between spatial resolution and measurement throughput needs to be well-thought-out. Several studies that were detecting disease in bigger scales are (Han 2013; Izzuddin et al. 2018; Huang et al. 2007).

In this study, Flavescence Dorée (FD) is classified in leaf scale. It is a bacterial grapevine disease caused by phytoplasma, spread in many counties in Europe (Chuche, Thiéry 2014). The symptoms of the disease start to appear in late summer and remain visible until mid-autumn. Typical transformations include white variety leaves turning into yellow colour nearby leaf veins and red caste leaves turning into dark red colour, inbetween leaves' veins spaces. Progressively, they curl into triangle shapes in both castes (Albetis et al. 2017). However, the goal of this study is to use a ML approach and evaluate selected classifiers based on their performance in classification of FD, even in a stage where no visible symptoms are present. Two Vinhão leaves with no visible symptoms of the disease can be seen in Figure 1, in which one of them is actually infected with FD (confirmed by laboratory tests).

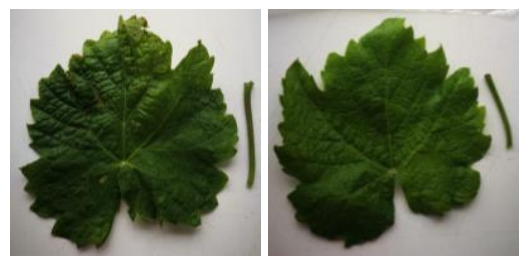

Figure 1. Vinhão FD negative leaf (left) and Vinhão FD positive leaf (right)

Machine learning (ML) approach demonstrated to be very useful to handle hyperspectral image analysis, mainly because of its capability to organize the relationship between the reflectance values and desired information while being robust against the noise and uncertainties in spectral and ground truth measurements (Gewali et al., 2019). This fact was demonstrated in a wide variety of studies (Preidl \& Doktor, 2011; Schneider et al., 2010; Verrelst et al.,2012), where in ML approach outperformed the traditional methods. Generally better that classical method such as spectral matching, ML can also handle a spectral and ground truth variability and noise (Schneider et al. 2010). This combination is booming in precision plant protection because number of applications shown promise for early disease detection (Golhani et al., 2018). However, main disadvantages include: highly dependency on patterns of variables, and features to be extracted, as well as the need for some classifiers to be trained several times before real application (Zhang et al. 2015).

\section{MATERIAL AND METHODS}

\subsection{Dataset}

\subsubsection{Data collection}

The leaves were collected in three campaigns, carried out in July, August and October of 2018, in which visual symptoms of the disease were picked. Campaigns took place in Minho region, Portugal (see Figure 2). The three campaigns were planned to acquire samples that would capture the behaviour of the disease in different stages of the development.

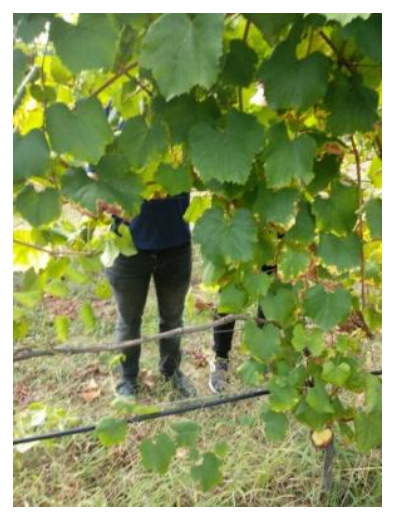

Figure 2. Vine leaves collection

The collection was done from two vine varieties: Vinhão and Loureiro, from red and white cultivars, respectively. Collected leaves were examined by a Nested polymerase chain reaction (Pelt-Verkuil et al., 2008) in laboratory environment and the presence of FD was tested.

The leaves were also scanned by Headwall Micro-Hyperspec VNIR E-Series sensor (Headwall 2017): a hyperspectral pushbroom scanner, combined with the Hyperspec STARTER KIT (Headwall 2007). This sensor was operating attached to a fixed laboratory table. To acquire imagery cubes, a linear push to make the device slide over the table was required. MicroHyperspec VNIR E-Series sensor has 272 spectral bands ranging between the visible light and a part of the near infrared region, more specifically, from $400-1000 \mathrm{~nm}$. To get a pure light reflectance signature for each image, each capture was matched with a previous light calibration procedure involving white and pure darkness light levels.

Approximately 80 leaves samples were collected per caste in each campaign. However, only healthy leaves and FD positive leaves with no other disease detected in laboratory examination were selected for the dataset.

The number of leaves that were used for creating the dataset can be seen in Table 1 .

\begin{tabular}{|l|r|r|r|r|}
\hline & \multicolumn{2}{|c|}{ Vinhão } & \multicolumn{2}{c|}{ Loureiro } \\
\hline Campaign & Negative & \multicolumn{1}{|l|}{ Positive } & Negative & \multicolumn{1}{l|}{ Positive } \\
\hline July & 22 & 6 & 14 & 12 \\
\hline August & 19 & 6 & 16 & 10 \\
\hline October & 25 & 10 & 23 & 7 \\
\hline
\end{tabular}

Table 1. Number of FD positive and FD negative leaves in each campaign used for a dataset

\subsubsection{Pre-processing}

After the samples collection, a pre-processing of the data was carried out. Acquired samples were in raw data cube format requiring calibration, which was done in special SpectralView software, a part of the hyperspectral sensor package. Afterwards, the background from hyperspectral image was removed by applying Spectral Angle Mapper (SAM) algorithm, through the calculation of spectral angle error, in radians, between two vectors, background and leaf pixels.

\subsubsection{Sample extraction}

Each hyperspectral leaf sample was composed of several pixels ( = 250000 pixels), each one characterized by a spectral signature. Due to the lack of positional information about leaf area disease incidence, two masks for selecting the spectral reflectance were used (see Figure 3). First mask (Figure 3 - left) represented the whole leaf. To reduce the computational burden, 
10000 spectral signatures representing one leaf were randomly selected for creating the first dataset. Under the orientations of plant science experts, masks around the (low) midrib - nearby leafstalk - was created (Figure 3 - right). Having in mind data balancing, 100 randomly selected spectral signatures per leaf was used for creating a second dataset.

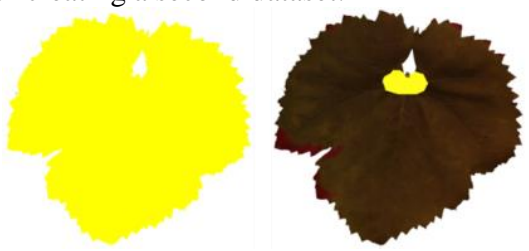

Figure 3. Masks for dataset production: left part shows the whole leafbased mask; at right, is an example depicting the low midrib-based mask

\subsection{Selected classifiers}

Classifiers can be viewed as labelling learning systems. They make few presumptions for classification without any prior knowledge of the data pattern. The goal of a classifier in this study is to estimate the presence of FD disease on vine leaves by analysing reflectance signatures, considering a classification problem. The first classifier was Logistic Regression (LR). This popular classifier is known for being suitable for binary classification problems (Hoffman 2019). Linear discriminant analysis (LDA) is a supervised dimensionality reducing method that finds directions that maximally separate the different classes while minimizing the spread within one class (Fisher 1936; Tavernier et al. 2019). Quadratic discriminant analysis (QDA) is an univariate statistical method. In QDA it is assumed that the measurements in each class have normal distribution, disregarding that the covariance of each class is the same (Eker et al., 2015). Multi-layer Perceptron (MLP) is a supervised learning algorithm that resorts to backpropagation for training. It can distinguish non-linearly separable data (Van Der Malsburg 1986). Naive Bayes (NB) is simple probabilistic classifiers based on applying Bayes' theorem with strong (naive) independent assumptions between the features (Maron 1961). Random forests classifier (RF) is a meta estimator that fits a number of decision tree classifiers on a meta estimator that fits a number of decision tree classifiers on more than one subsamples of the dataset and uses averaging to improve the predictive accuracy and control over-fitting (Breiman 2001). Decision trees classifier (DT) is a predictive modelling approach that uses a decision tree to go from observations about an item to conclusions about its target value (Quinlan 1986). K-Nearest Neighbours (KNN) is one of the most known and used methods for supervised pattern recognition. (Coomans and Massart 1982). The sample is classified by a plurality vote of its neighbors and is assigned to the class that is most common among its $k$ nearest neighbors (Altman 1992).

\section{RESULTS}

Results were calculated from two different dataset that were created by the selected masks (see Figure 3).

\subsection{Predictions within campaign}

First predictions were estimated for unseen samples from the same campaign as training samples. Because of the limited number of samples and to avoid biased estimate, standard kcross-validation resampling procedure was applied. The parameter $\mathrm{k}$ was selected to 10 , which split the training dataset into 10 subsets. This approach provides a reasonable estimation of a classifier performance on unseen samples.

In, Table 2, a prediction accuracies of selected classifiers, see Chapter 2.2, for Vinhão caste are presented.

\begin{tabular}{|l|r|r|r|r|r|r|}
\hline & \multicolumn{2}{|c|}{ October } & \multicolumn{2}{c|}{ August } & \multicolumn{2}{c|}{ July } \\
\hline & Leaf & Middle & Leaf & Middle & Leaf & Middle \\
\hline LR & $82 \%$ & $83 \%$ & $68 \%$ & $69 \%$ & $83 \%$ & $85 \%$ \\
\hline LDA & $80 \%$ & $84 \%$ & $72 \%$ & $67 \%$ & $81 \%$ & $85 \%$ \\
\hline QDA & $63 \%$ & $81 \%$ & $77 \%$ & $76 \%$ & $80 \%$ & $88 \%$ \\
\hline MLP & $85 \%$ & $84 \%$ & $72 \%$ & $73 \%$ & $89 \%$ & $86 \%$ \\
\hline NB & $71 \%$ & $53 \%$ & $68 \%$ & $53 \%$ & $72 \%$ & $75 \%$ \\
\hline RF & $85 \%$ & $80 \%$ & $77 \%$ & $76 \%$ & $81 \%$ & $87 \%$ \\
\hline DT & $87 \%$ & $83 \%$ & $77 \%$ & $72 \%$ & $71 \%$ & $86 \%$ \\
\hline KNN & $76 \%$ & $80 \%$ & $67 \%$ & $70 \%$ & $85 \%$ & $86 \%$ \\
\hline
\end{tabular}

Table 2. Prediction accuracies for Vinhão in three campaigns

Best results in October campaign were achieved by MLP and DT. The former achieved $85 \%$ accuracy when using samples from the whole leaves and $84 \%$ when using samples from middle part of the leaf, while the latter achieved $87 \%$ and $83 \%$ using same datasets. Worst results can be spotted for NB and QDA when using the dataset composed of middle area spectral signatures. Worst classification accuracies, comparatively to October, were obvious in August campaign, not reaching over $77 \%$. In July campaign, the overall accuracies were higher than in August, and similar to October.

The results for Loureiro can be seen in Table 3. From the table is clear that classification accuracies in October calculated by using second dataset were higher for all the classifiers than by using samples from the whole leaf. LR and LDA achieved $94 \%$ of accuracy. However, the same trend was not obvious in August campaign. Top- 2 classifiers were again LR and LDA but achieved by using samples from the whole leaf. Very poor results were achieved in July, where most of the classifiers did not reach $60 \%$ of accuracy.

\begin{tabular}{|l|c|r|r|r|r|r|}
\hline & \multicolumn{2}{|c|}{ October } & \multicolumn{2}{c|}{ August } & \multicolumn{2}{|c|}{ July } \\
\hline & Leaf & Middle & Leaf & Middle & Leaf & Middle \\
\hline LR & $92 \%$ & $94 \%$ & $73 \%$ & $71 \%$ & $53 \%$ & $52 \%$ \\
\hline LDA & $89 \%$ & $94 \%$ & $87 \%$ & $72 \%$ & $56 \%$ & $49 \%$ \\
\hline QDA & $69 \%$ & $88 \%$ & $60 \%$ & $68 \%$ & $73 \%$ & $56 \%$ \\
\hline MLP & $92 \%$ & $93 \%$ & $72 \%$ & $70 \%$ & $55 \%$ & $51 \%$ \\
\hline NB & $81 \%$ & $86 \%$ & $65 \%$ & $55 \%$ & $51 \%$ & $57 \%$ \\
\hline RF & $86 \%$ & $91 \%$ & $52 \%$ & $67 \%$ & $42 \%$ & $56 \%$ \\
\hline DT & $83 \%$ & $93 \%$ & $57 \%$ & $65 \%$ & $56 \%$ & $56 \%$ \\
\hline KNN & $88 \%$ & $93 \%$ & $55 \%$ & $66 \%$ & $48 \%$ & $55 \%$ \\
\hline
\end{tabular}

Table 3. Prediction accuracies for Loureiro in three campaigns

\subsection{Backwarding predictions among different campaigns}

Predictions among different campaigns in a backward manner i.e., from later to earlier field missions - were carried out. Prediction accuracies in a previous campaign for Vinhão can be seen in Table 4.

\begin{tabular}{|l|l|l|l|l|l|l|}
\hline & \multicolumn{2}{|c|}{$\begin{array}{c}\text { October to } \\
\text { August }\end{array}$} & \multicolumn{2}{c|}{ October to July } & \multicolumn{2}{c|}{ August to July } \\
\hline & Leaf & Middle & Leaf & Middle & Leaf & Middle \\
\hline
\end{tabular}




\begin{tabular}{|l|l|l|l|l|l|l|}
\hline LR & $80 \%$ & $82 \%$ & $68 \%$ & $74 \%$ & $81 \%$ & $83 \%$ \\
\hline LDA & $81 \%$ & $83 \%$ & $68 \%$ & $74 \%$ & $82 \%$ & $76 \%$ \\
\hline QDA & $54 \%$ & $86 \%$ & $40 \%$ & $74 \%$ & $56 \%$ & $88 \%$ \\
\hline MLP & $85 \%$ & $82 \%$ & $68 \%$ & $73 \%$ & $85 \%$ & $88 \%$ \\
\hline NB & $15 \%$ & $31 \%$ & $28 \%$ & $50 \%$ & $61 \%$ & $53 \%$ \\
\hline RF & $83 \%$ & $86 \%$ & $76 \%$ & $74 \%$ & $85 \%$ & $87 \%$ \\
\hline DT & $79 \%$ & $80 \%$ & $56 \%$ & $74 \%$ & $73 \%$ & $79 \%$ \\
\hline KNN & $84 \%$ & $79 \%$ & $76 \%$ & $71 \%$ & $81 \%$ & $75 \%$ \\
\hline
\end{tabular}

Table 4. Prediction accuracies into previous campaign for Vinhão

Best prediction accuracies in Vinhão from October to August were achieved by RF with $86 \%$ using the second dataset and by MLP with $85 \%$ using the first dataset. Very poor performance was achieved by NB. Best results from October to July were achieved with RF and MLP; the latter had performances similar to LR and LDA. In predictions from August to July best scores were clearly achieved by RF and MLP.

In Table 5. are presented predictions results to previous campaigns for Loureiro

\begin{tabular}{|l|r|r|c|r|r|r|}
\hline & \multicolumn{2}{|c|}{$\begin{array}{c}\text { October to } \\
\text { August }\end{array}$} & \multicolumn{2}{c|}{ October to July } & \multicolumn{2}{c|}{ August to July } \\
\hline & Leaf & Middle & Leaf & Middle & Leaf & Middle \\
\hline LR & $69 \%$ & $72 \%$ & $54 \%$ & $56 \%$ & $54 \%$ & $53 \%$ \\
\hline LDA & $58 \%$ & $71 \%$ & $54 \%$ & $57 \%$ & $46 \%$ & $49 \%$ \\
\hline QDA & $58 \%$ & $65 \%$ & $54 \%$ & $56 \%$ & $65 \%$ & $53 \%$ \\
\hline MLP & $65 \%$ & $71 \%$ & $54 \%$ & $56 \%$ & $54 \%$ & $52 \%$ \\
\hline NB & $42 \%$ & $59 \%$ & $58 \%$ & $58 \%$ & $42 \%$ & $54 \%$ \\
\hline RF & $65 \%$ & $62 \%$ & $54 \%$ & $57 \%$ & $54 \%$ & $55 \%$ \\
\hline DT & $65 \%$ & $67 \%$ & $58 \%$ & $56 \%$ & $42 \%$ & $51 \%$ \\
\hline KNN & $73 \%$ & $64 \%$ & $54 \%$ & $56 \%$ & $62 \%$ & $53 \%$ \\
\hline
\end{tabular}

Table 5. Prediction accuracies into previous campaign for Loureiro

Overall prediction accuracies to previous campaign in Loureiro caste were low. Highest scores were achieved in prediction from October to August, by LR, LDA, MLP and KNN, with about $70 \%$. Prediction from October to July and from August to July did not surpass $65 \%$.

\section{CONCLUSION}

The presented results showed that classification of FD using hyperspectral vine leaf images and ML approach is possible with reasonable accuracies while predicting within a same campaign, especially in the latest (October), where the disease is most developed. In Vinhão variety, the best classifiers were DT with $87 \%$ calculated using the samples from whole leaf, and MLP with $85 \%$ calculated from the same dataset. In Loureiro variety best performance was achieved by LR and LDA with 94\% using the low midrib-based dataset. Minor differences were observed between mentioned datasets, despite the discrepancy of the number of samples collected for each dataset (1000x wider). The greatest difference can be identified in the results associated to the earliest campaign (July), where the prediction accuracies in Loureiro variety were poor. Several hypotheses might be considered as eventual justifications for such observation. One of them regards to disease's early stage in that particular campaign, which smooths characteristics while posing challenges to classification between "healthiness" and "infection", in a spectral perspective. Another one, can be related to representativity issues of the selected samples for training, more concretely, the uncertainty factor associated to pixels' FD labelling on leaves confirmed as infected through laboratory tests, a potential deception inducer for the classifier. Selecting all the samples from a leaf would not tackle this issue either, due to the eventual increased number of wrong labels that would be in use. Finally, the "curse of dimensionality", also known as Hughes phenomenon, caused by the high number of features and the limited number of training samples might be in the origin of the lately mentioned poor results. In that sense, the success of supervised ML is, of course, intimately related to dataset labelling consistency and correctness. Unsupervised learning might be complementary to this task, enabling userbased categorization of organized data clusters.

Among predictions in Vinhão variety, campaigns prediction backwarding from October to August was best evaluated by QDA with $86 \%$, using the low midrib-based dataset. Best prediction from October to earliest campaign in July was achieved by $\mathrm{KNN}$ with $76 \%$, using the whole leaf-based dataset. In August to July prediction backwarding, best performances were achieved by MLP and QDA with $88 \%$, calculated from the low midrib-based dataset.

Prediction backwarding in Loureiro had diverse outcomes. From October to August, the best classifier was KNN with 73\%, using the whole leaf-based dataset. However, regarding October to July predictions, none of the classifiers reached over 58\%. In August to July predictions, only two classifiers reached over 62\%: KNN and QDA.

To achieve higher prediction accuracies for the task of plant disease detection, more robust and verified datasets of healthy and diseased leaves are needed. In future work, dimensionality reduction techniques will be explored, due to the redundancy induced by the hundreds of contiguous spectral bands composing hyperspectral imagery.

\section{ACKNOWLEDGEMENTS}

This work was financed by the European Regional Development Fund (ERDF) through the Operational Programme for Competitiveness and InternationalisationCOMPETE 2020 under the PORTUGAL 2020 Partnership Agreement, and through the Portuguese National Innovation Agency (ANI) as a part of project "PARRA-Plataforma integrAda de monitoRização e avaliação da doença da flavescência douRada na vinhA" ( $\left.\mathrm{N}^{\circ} 3447\right)$ and ERDF and North 2020 - North Regional Operational Program, as part of project "INNOVINE \& WINE-Vineyard and Wine Innovation Platform" (NORTE-01-0145-FEDER-000038).

\section{REFERENCES}

Adão, Telmo, Jonáš Hruška, Luís Pádua, et al 2017 Hyperspectral Imaging: A Review on UAV-Based Sensors, Data Processing and Applications for Agriculture and Forestry. Remote Sensing 9(11): 1110.

Akila, $\mathrm{M}, \quad$ and $\mathrm{P}$ Deepan 2018 Detection and Classification of Plant Leaf Diseases by Using Deep Learning Algorithm. International Journal of Engineering Research 6(07): 5.

Albetis, Johanna, Sylvie Duthoit, Fabio Guttler, et al. 2017 Detection of Flavescence Dorée Grapevine Disease 
Using Unmanned Aerial Vehicle (UAV) Multispectral Imagery. Remote Sensing 9(4): 308.

Altman,

N.

$\mathrm{S}$

1992 An Introduction to Kernel and Nearest-Neighbor Nonparametric Regression. The American Statistician 46(3): 175-185.

Breiman, Leo

2001 Random Forests. Machine Learning 45(1): 5-32.

Chen, Tingting, Jialei Zhang, Yong Chen, Shubo Wan, and Lei Zhang

2019 Detection of Peanut Leaf Spots Disease Using Canopy Hyperspectral Reflectance. Computers and Electronics in Agriculture 156: 677-683.

Chuche, Julien, and Denis Thiéry 2014 Biology and Ecology of the Flavescence Dorée Vector Scaphoideus Titanus: A Review. Agronomy for Sustainable Development 34(2): 381-403.

Coomans, D., and D. L. Massart 1982 Alternative K-Nearest Neighbour Rules in Supervised Pattern Recognition: Part 1. k-Nearest Neighbour Classification by Using Alternative Voting Rules. Analytica Chimica Acta 136: $15-27$.

Eker, Arif Mert, Mehmet Dikmen, Selim Cambazoğlu, Şebnem H. B. Düzgün, and Haluk Akgün 2015 Evaluation and Comparison of Landslide Susceptibility Mapping Methods: A Case Study for the Ulus District, Bartın, Northern Turkey. International Journal of Geographical Information Science 29(1): 132-158.

Fisher, $\mathrm{R}$. A.

1936 The Use of Multiple Measurements in Taxonomic Problems. Annals of Eugenics 7(2): 179-188.

Gewali, Utsav B, Sildomar T Monteiro, and Eli Saber 2019 Machine Learning Based Hyperspectral Image Analysis: A Survey: 46.

Golhani, Kamlesh, Siva K. Balasundram, Ganesan Vadamalai, and Biswajeet Pradhan 2018 A Review of Neural Networks in Plant Disease Detection Using Hyperspectral Data. Information Processing in Agriculture 5(3): 354-371.

Han,

2013 Airborne Hyperspectral Imaging Based Citrus Greening Disease Detection Using Different Dimension Reduction Methods. In 2013 ASABE Annual International Meeting Pp. 1-12. American Society of Agricultural and Biological Engineers. http://elibrary.asabe.org/abstract.asp?aid $=44124 \& \mathrm{t}=3 \&$ dabs $=\mathrm{Y}$ \&redir=\&redirType=, accessed July 28, 2019.

Headwall 2007
https://www.polytec.com/fileadmin/d/Photonik/Optische_Syste . me/kameras-und-

kamerasysteme/PH_WL_Headwall_Hyperspec_StarterKit_2007 .pdf.
2017 Headwall Product Data Sheet https://cdn2.hubspot.net/hubfs/145999/docs/MicroHyperspec_2017.pdf?t=1502897661972.

Hoffman, Julien I. E. 2019 Chapter 33 - Logistic Regression. In Basic Biostatistics for Medical and Biomedical Practitioners (Second Edition). Julien I. E. Hoffman, ed. Pp. 581-589. Academic Press.

http://www.sciencedirect.com/science/article/pii/B97801281708 47000334, accessed July 26, 2019.

Huang, Wenjiang, David W. Lamb, Zheng Niu, et al. 2007 Identification of Yellow Rust in Wheat Using In-Situ Spectral Reflectance Measurements and Airborne Hyperspectral Imaging. Precision Agriculture 8(4): 187-197.

Izzuddin, M A, M N Nisfariza, B Ezzati, et al. 2018. https://doi.org/10.21894/jopr.2018.0037.

Lowe, Amy, Nicola Harrison, and Andrew P French 2017 Hyperspectral Image Analysis Techniques for the Detection and Classification of the Early Onset of Plant Disease and Stress. Plant Methods 13. https://www.ncbi.nlm.nih.gov/pmc/articles/PMC5634902/, accessed January 9, 2018.

Mahlein,

Anne-Katrin 2015 Plant Disease Detection by Imaging Sensors Parallels and Specific Demands for Precision Agriculture and Plant Phenotyping. Plant Disease 100(2): 241-251.

Mahlein, Anne-Katrin, Ulrike Steiner, Christian Hillnhütter, Heinz-Wilhelm Dehne, and Erich-Christian Oerke 2012 Hyperspectral Imaging for Small-Scale Analysis of Symptoms Caused by Different Sugar Beet Diseases. Plant Methods 8(1): 3 .

Maron, M. E. 1961 Automatic Indexing: An Experimental Inquiry. J. ACM 8(3): 404-417.

Martinelli, Federico, Riccardo Scalenghe, Salvatore Davino, et al.

2015 Advanced Methods of Plant Disease Detection. A Review. Agronomy for Sustainable Development 35(1): 1-25.

Pelt-Verkuil, Elizabeth van, Alex van Belkum, and John P. Hays

2008 Principles and Technical Aspects of PCR Amplification. Springer Science \& Business Media.

Popp, József, Károly Pető, and János Nagy 2013 Pesticide Productivity and Food Security. A Review. Agronomy for Sustainable Development 33(1): 243-255.

Preidl, Sebastian, and Daniel Doktor 2011 Comparison of Radiative Transfer Model Inversions to Estimate Vegetation Physiological Status Based on Hyperspectral Data. https://www.infona.pl//resource/bwmeta1.element.ieee-art000006080936, accessed July 28, 2019.

Quinlan, J. $\mathrm{R}$. 1986 Induction of Decision Trees. Machine Learning 1(1): 81-106. 
Santesteban,

Luis

2019 Precision Viticulture and Advanced Analytics. A Short Review. Food Chemistry 279: 58-62.

Savary, Serge, Andrea Ficke, Jean-Noël Aubertot, and Clayton Hollier

2012 Crop Losses Due to Diseases and Their Implications for Global Food Production Losses and Food Security. Food Security 4(4): 519-537.

Schneider, S., A. Melkumyan, R. J. Murphy, and E. Nettleton 2010 Gaussian Processes with OAD Covariance Function for Hyperspectral Data Classification. https://www.infona.pl//resource/bwmeta1.element.ieee-art000005670066, accessed July 28, 2019.

Tavernier, Joris, Jaak Simm, Karl Meerbergen, et al. 2019 Fast Semi-Supervised Discriminant Analysis for Binary Classification of Large Data Sets. Pattern Recognition 91: 86-99.

Thomas, Stefan, Matheus Thomas Kuska, David Bohnenkamp, et

2017 Benefits of Hyperspectral Imaging for Plant Disease Detection and Plant Protection: A Technical Perspective SpringerLink.

https://link.springer.com/article/10.1007\%2Fs41348-017-01246, accessed July 27, 2019.

Van Der Malsburg, C. 1986 Frank Rosenblatt: Principles of Neurodynamics: Perceptrons and the Theory of Brain Mechanisms. In Brain Theory. Günther Palm and Ad Aertsen, eds. Pp. 245-248. Springer Berlin Heidelberg.

Verrelst, Jochem, Luis Alonso, Gustavo Camps-Valls, Jesús Delegido, and José F. Moreno 2012 Retrieval of Vegetation Biophysical Parameters Using Gaussian Process Techniques. IEEE Transactions on Geoscience and Remote Sensing 50: 1832-1843.

Virlet, Nicolas, Kasra Sabermanesh, Pouria Sadeghi-Tehran, and Malcolm J. Hawkesford 2017 Field Scanalyzer: An Automated Robotic Field Phenotyping Platform for Detailed Crop Monitoring. Functional Plant Biology 44(1): 143-153.

Walter, Achim, Frank Liebisch, and Andreas Hund 2015 Plant Phenotyping: From Bean Weighing to Image Analysis. Plant Methods 11(1): 14.

Xie, Chuanqi, Yongni Shao, Xiaoli $\mathrm{Li}$, and Yong $\mathrm{He}$ 2015 Detection of Early Blight and Late Blight Diseases on Tomato Leaves Using Hyperspectral Imaging. Scientific Reports

https://www.ncbi.nlm.nih.gov/pmc/articles/PMC4647840/, accessed July 27, 2019.

Zhang, Baohua, Wenqian Huang, Chaopeng Wang, et al. 2015 Computer Vision Recognition of Stem and Calyx in Apples Using Near-Infrared Linear-Array Structured Light and 3D Reconstruction. Biosystems Engineering 139: 25-34.

Zhao, Yan-Ru, Xiaoli Li, Ke-Qiang Yu, Fan Cheng, and Yong $\mathrm{He}$
2016 Hyperspectral Imaging for Determining Pigment Contents in Cucumber Leaves in Response to Angular Leaf Spot Disease. Scientific Reports 6. https://www.ncbi.nlm.nih.gov/pmc/articles/PMC4901261/, accessed July 27, 2019.

Zhou, Rui-Qing, Juan-Juan Jin, Qing-Mian Li, et al. 2019 Early Detection of Magnaporthe Oryzae-Infected Barley Leaves and Lesion Visualization Based on Hyperspectral Imaging. Frontiers in Plant Science 9. https://www.frontiersin.org/articles/10.3389/fpls.2018.01962/fu 11 , accessed July 27, 2019. 